\title{
Role of High Resolution Ultrasound and Magnetic Resonance Imaging In Assessment of the Posterior Ankle Tendons Lesions
}

\author{
Hesham M. Farouk $^{\mathrm{a}}$, Ahmed H. Saber ${ }^{\mathrm{a}}$, Alaa-Eldin A. Mohamed ${ }^{\mathrm{b}}$
}

\begin{abstract}
a Department of radiology, Benha faculty of medicine, Banha University, Egypt. b Department of radiology, Ghamra Military Hospital Professor at Military Medical Academy, Cairo, Egypt.

Correspondence to: Ahmed H. Saber, Department of radiology, Benha faculty of medicine, Banha University, Egypt.
\end{abstract}

Email:

dr.hamza3111@gmail.com

Received: 2 August 2021

Accepted: 14 December 2021

\begin{abstract}
Objectives: Ankle tendons may be affected by a variety of pathologic conditions, including trauma and overuse, inflammatory and metabolic disorders, or infections. Ultrasound is a very sensitive means of detecting tendinous pathology because of its spatial resolution and its comparative and dynamic capabilities. Its wide availability makes it the preferred first-line imaging modality in cases of ankle pain. Knowledge of the normal anatomy, the clinical symptoms and the setting may help in different diagnoses. Aim of study: this study aims to highlight the role of high-resolution Ultrasound Doppler and MRI in assessing posterior ankle tendons lesions. Patients and methods: Our study is a cross-sectional study, included 34 patients, referred to our Radiology Department at Benha University Hospital with unilateral painful ankles between 2020 and 2021. The study was accepted by the local ethical committee. Results: Ultrasound and MRI revealed nearly the same results in the evaluation of tendon abnormalities. Our study included 53
\end{abstract} tendinous pathological entities detected by MR (standard reference imaging modality) which classified into; degenerative (17 lesions), inflammatory (25 lesions) and tears (partial: 7 or complete: 4 lesions). Two lesions are missed by Ultrasound. Conclusion: Ultrasonography is an accurate and sensitive modality in assessing the tendons around the ankle joint and can be used either as a primary tool of investigation or as a complementary tool with MRI and even in some cases may be used as a final method of diagnosis without the need for repeating both techniques.

Keywords: MRI, US, Posterior ankle tendons lesions.

\section{Introduction:}

The ankle is often the most commonly usually from an inversion injury in a twisted injured region of the lower extremity, or sprained ankle ${ }^{[1]}$. The posterior ankle 
tendons include Achilles tendon, tibialis posterior, flexor digitorum longus and flexor halluces longus. The Achilles tendon is the thickest and strongest tendon in the man. Its length ranges from 110 to $260 \mathrm{~mm}$ (average is $150 \mathrm{~mm}$ ) and it has a broad shape with an average width of $(68 \mathrm{~mm})$. Its origin from the aponeuroses of the medial, lateral and soleus gastrocnemius muscles (triceps surae) and its insertion is into the posterior calcaneal tuberosity ${ }^{[2]}$.

The ankle tendons disorders can be classified into two main groups. The first group consists of different types of tendinopathy that are not related to mechanical stress, such as inflammatory, infective, neoplastic and metabolic lesions. The second group includes mechanical tendipathies that are related to mechanical damage specific for each tendon ${ }^{[3]}$. The most common pathologies of the posterior compartment are the pathologies related to the Achilles tendon which can be either acute injury or chronic pathology. Acute injury is mostly related to sports. Chronic pathology has a chronic background and also called tendinopathy ${ }^{[4]}$.

Both ultrasound (US) and magnetic resonance imaging (MRI) can provide excellent morphological information and can distinguish the various structural abnormalities that can be found along the tendon ${ }^{[5]}$. The main advantage of ultrasonography over MRI is its easy availability, repeatability, comparison with the contralateral side, low cost and ease of examination with dynamic capability. Infections can be detected with USG, but related bony involvement is difficult to evaluate. MRI offers an advantage of distinguishing bony involvement with high accuracy and gives an excellent image resolution for assessing the tendon pathologies ${ }^{[6]}$. The above all discussion embarks on the importance of both modalities in tendon pathology. Ultrasonography to be the most valid and widely used tool due to its easy accessibility but MRI is pivotal for diagnosis and prognosis.

\section{Aim of study}

This study aims to highlight the role of highresolution ultrasound Doppler and MRI in the assessment of posterior ankle tendons lesions.

\section{Patients and methods}

This is a cross-sectional study. The study was carried on 34 patients who were referred to the Radiology Department of 
Benha University from March 2020 to March 2021.

The approval of the ethical committee at Benha university was granted before beginning of work.

All of the patients were subjected to:

- Full history taking.

- Full clinical examination.

- Ultrasound examination.

- MRI examination.

\section{Inclusion criteria:}

Patients complaining of posterior heel pain, discomfort, stiffness, swelling, limitation of movements, diffuse thickening of the soft tissue around posterior aspect of the ankle, ecchymotic ankle either spontaneous, related to trauma or rheumatological disorder.

\section{Exclusion criteria:}

Patients contraindicated with MRI examinations such as patients who have cochlear implants, cardiac pacemakers, aneurysm clip, and severely claustrophobic patients.

Ultrasound examination will be performed using the same ultrasound system (LOGIQ P6 PRO) and the same linear transducer (7 $12 \mathrm{MHz}$ ). All patients had standardized ultrasound examinations of both ankles for comparison. For examination of the Achilles tendon; the patient is positioned prone with the foot hanging freely over the edge of the examination table and excess gel was used. We perform a survey scan, in transverse, from its myotendinous junction to its calcaneal insertion and we can measure the thickness in the short axis to avoid overestimation of the tendon thickness in the long-axis image. Rotate into longitudinal and examine for thickening and integrity. After B-mode examination, Interrogation of color and power Doppler was done to detect the vascularity. At the same time, we assess the retro-calcaneal bursae and Kager's fat pad. Dynamic evaluation is achieved by actively plantar flexing and dorsiflexing the ankle to evaluate suspected tendon tears. The medial aspect of the ankle was scanned after placing the patient in either the right or left lateral oblique position. The posterior tibial tendons, flexor digitorum longus, and flexor hallucis longus are examined in both longitudinal and transverse planes. For identification and evaluation of the flexor hallucis longus, the patient's great toe is gently flex and extend (dynamic maneuvers).

MR examination was done for all patients using Magnetom symphony, syngo, $1.5 \mathrm{~T}$ 
machine. MRI examination will be done using the standard circular extremity coil. The patient is positioned supine with the foot and ankle within the circular extremity coil, the foot was dorsiflexed with the dorsal aspect of the foot at right angle to the examination couch, the leg was immobilized in a comfortable position by pads. Scout T1 weighted images were taken first followed by axial, sagittal T2 weighted images (TR: 2000-4000 ms, TE: 80-100 ms), axial and coronal T1 weighted images (TR: 400-600 ms, TE: 15-25 ms), sagittal and coronal STIR (TR: 3550, TE: 34, TI: $150 \mathrm{~ms}$ ), axial T1 weighted images with fat suppression (TR: $775 \mathrm{~ms}$, TE: $12 \mathrm{~ms}$ ), slice thickness 4 $\mathrm{mm}$, interslice gap 2-3 mm, matrix size 256 X 256 pixels, FOV $16 \mathrm{~cm}$ and covering the whole length of the Ankle joint. The Achilles tendon was best assessed in the sagittal STIR image.

\section{$\underline{\text { Statistical methods }}$}

Analysis of data was done using Statistical Program for Social Science version 22 (SPSS Inc., Chicago, IL, USA). Quantitative variables were described in the form of mean and standard deviation. Qualitative variables were described as number and percent.

\section{Results}

This study included 34 patients who were referred to the Radiology Department of Benha University Hospital. All patients were subjected to full history taking, full clinical examination, US and MRI examinations. It included 29 male patients and 5 female patients with an age range from 14 to 75 years. All of the examined patients were suffering from posterior ankle pain. In 21 patients the right ankle was affected (62\%) and in 13 patients the left ankle was affected $(38 \%)$.

Our study included 53 tendinous pathological entities detected by MR which classified into; degenerative (Tendinosis: 17 lesions), inflammatory conditions (tenosynovitis: 25 lesions) and tendon tears (partial: 7 or complete: 4 lesions). In comparison to MRI which is considered the standard reference imaging modality in our study ultrasound diagnosed 51 lesions with distribution and classification as seen in tables (1, 2) and (Fig 1). The two missed lesions by ultrasound were one case of a partial tear of the Achilles tendon and one case of FHL tenosynovitis. 
As regarding the ability of ultrasound in the characterization of different tendon pathologies, we summarized our results in table (3).

Table (1): Distribution of tendinous pathological entities diagnosed by US in comparison to MRI in the study.

\begin{tabular}{lll}
\hline & $\begin{array}{l}\text { No. of Pathological entities } \\
\text { diagnosed by MRI }\end{array}$ & $\begin{array}{l}\text { No. of Pathological entities } \\
\text { diagnosed by US }\end{array}$ \\
\hline Achilles Tendon & 25 & 24 \\
Tibialis Posterior & 14 & 14 \\
Flexor Hallucis Longus & 10 & 9 \\
Flexor Digitorum Longus & 4 & 4 \\
Total & 53 & 51 \\
\hline
\end{tabular}

Table (2): Classification of tendinous pathologies diagnosed by US in comparison to MRI in the study.

\begin{tabular}{llll}
\hline Pathology & Diagnosed by MRI & Diagnosed by U/S \\
\hline 1. Achilles Tendon & & 11 \\
- Tendinosis & 11 & 4 \\
- Peritendinitis & 4 & 5 \\
- Partial tear & 6 & 4 \\
- Complete tear & 4 & \\
2. Tibialis Posterior & 2 & 2 \\
- Tendinosis & 11 & 11 \\
- Tenosynovitis & 1 & 1 \\
- Partial tear & & \\
3. Flexor Hallucis Longus & 10 & 9 \\
- Tenosynovitis & & 4 \\
4. Flexor Digitorum Longus & 4 & 51 \\
- Tenosynovitis & 53 & \\
Total & &
\end{tabular}


Benha medical journal, vol. 39, special issue (radiology), 2022

Table (3). Ability of ultrasound for identification and characterization of different tendon injuries.

\begin{tabular}{|c|c|c|c|c|c|c|}
\hline & & 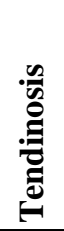 & 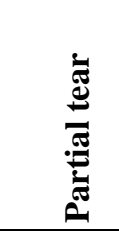 & & & 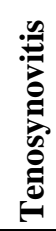 \\
\hline - Sensitivity & $100 \%$ & & $85.7 \%$ & $100 \%$ & $96 \%$ & \\
\hline - Specificity & $100 \%$ & & $100 \%$ & $100 \%$ & $100 \%$ & \\
\hline - + Ve predictive value & $100 \%$ & & $100 \%$ & $100 \%$ & $100 \%$ & \\
\hline - - Ve predictive value & $100 \%$ & & $99.2 \%$ & $100 \%$ & $98.7 \%$ & \\
\hline - Accuracy & $100 \%$ & & $99.3 \%$ & $100 \%$ & $99 \%$ & \\
\hline
\end{tabular}

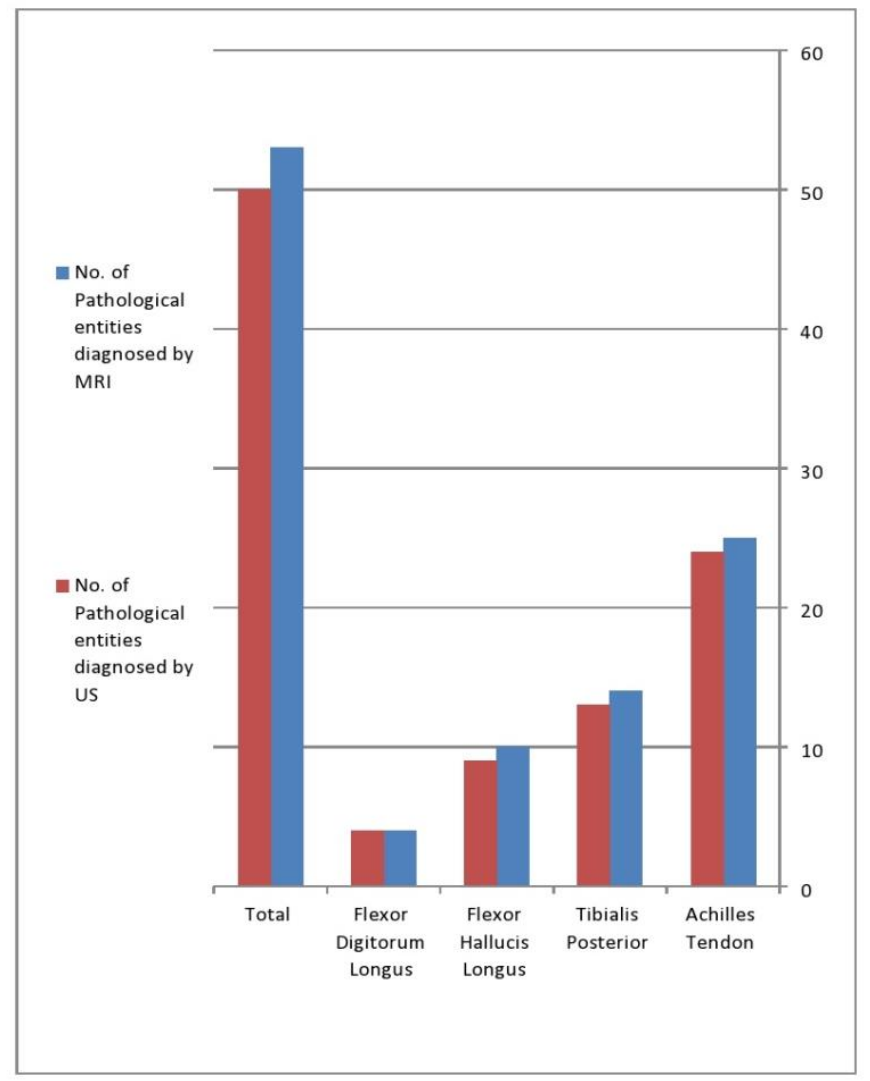

Figure (1), Distribution of tendinous pathological entities diagnosed by US in comparison to MRI in the study 


\section{Discussion}

The development of high-frequency high-resolution modern electronic transducers has increased the possibility of ultrasonography in the detection of normal small anatomic structures and the assessment of a variety of musculoskeletal disorders. These improvements along with economic factors and availability have resulted in renewed interest in using US as a diagnostic tool. As such, the efficacy of US has been compared with other modalities, in particular MRI, as a cost-effective imaging alternative ${ }^{[7]}$. This study included thirtyfour cases with posterior ankle pain to evaluate the role of high-resolution ultrasound Doppler and MRI in the assessment of posterior ankle tendons lesions.

The Achilles tendon is the most commonly injured ankle tendon. Our study coincides with that as $47 \%$ of all tendon injuries in our study implicated Achilles tendon ranging between tendinosis, partial tear, to complete tear. There were 25 cases of Achilles tendon injuries representing $47 \%$ of the tendinous injuries. $16 \%$ of these cases (4 cases) were presented with complete rupture of the Achilles tendon. Their ages ranged between 25 and 51 years. This is matched with other studies ${ }^{[8]}$ who reported that Achilles tendon ruptures are commonly affecting middleaged individuals and abnormal tendons. The rest of Achilles tendon injuries in our study were presented with partial tear, tendinosis, and paratenonitis representing 24\%, 44\% and $16 \%$ of the Achilles tendon injuries, respectively. In our study, the sensitivity, specificity and accuracy for US for Achilles tendon were 96\%, 100\% and 98\% respectively which coincident with the study done $2018^{[9]}$, where it was found that ultrasonography is effective in the differentiation of full versus partialthickness tears or tendinopathy, with a sensitivity, specificity and accuracy of $97 \%$, $96.4 \%$ and $98 \%$ respectively. In our study, ultrasonography succeeded to diagnose all Achilles tendon injuries tendinosis, partial tear, and complete tear similar to MRI excepting for only one case of partial tear.

The medial compartment tendons of the ankle were the most second tendons to be affected, the Tibialis posterior tendon was the most common one in our study. Our study included 14 Tibialis posterior tendon pathology were diagnosed by ultrasound and MRI. Our results were similar to the results achieved before ${ }^{[10]} \&{ }^{[8]}$ and where a 
correlation of ultrasound findings in Tibialis posterior tendon injuries with surgical findings was found and showed that ultrasound allowed correct diagnosis in all of the 14 cases of tendon diseases, including tendinosis, tenosynovitis, partial and complete tear. Ultrasonography was showed $100 \%$ sensitivity with the ability to detect the Tibialis posterior tendon injuries identified at the magnetic resonance imaging.

Of the remaining medial ankle tendons, the Flexor Digitorum Longus tendon is rarely affected by pathologic changes but can be examined by ultrasonography. Our study included four cases of FDL tenosynovitis which was diagnosed by US and approved by MRI study which represents $12 \%$ of all cases with sensitivity $100 \%$. In this study, ten patients with Flexor Hallucis Longus tenosynovitis were diagnosed. Only one case was missed by ultrasonography which confirmed by magnetic resonance imaging with sensitivity $90 \%$, specificity $100 \%$ and accuracy $97 \%$, this agreed with a study done $2013^{[11]}$ where it was stated that the flexor digitorum longus tendon is rarely affected by tenosynovitis and the flexor hallucis longus tendon is more commonly affected.
Regarding posterior impingement syndrome was reported in 3 cases in our study with prominent steida process being the commonest etiological factor, this agreed with other research carried out in $2016^{[12]}$ where it was stated that prominent steida process is the main osseous cause of the posterior ankle impingement.

Ultrasonography is a sensitive, low cost and rapid diagnostic modality yet it is highly operator-dependent.

\section{Conclusion:}

Ultrasound is an excellent cost-benefit widely available imaging modality that has high spatial resolution making it a helpful tool in diagnosing musculoskeletal ankle disorders mainly when evaluation soft tissue structures and extremely valuable when a focused evaluation is needed for a soft tissue structure or precisely examining the region of symptoms. Ultrasonography with the advent of high-resolution linear array as a dynamic, rapid and inexpensive imaging tool and with high accuracy as magnetic resonance imaging can be used as a first-line diagnostic modality in patients with acute tendons injuries. Ultrasound examination is also valuable in assessing ankle tendons injuries when metallic artifacts would limit imaging with MRI or CT. Magnetic 
resonance imaging is an excellent technique for those cases where the diagnosis is uncertain or cannot be confirmed by ultrasonography and shows the extent of the lesion especially when surgical interference is planned. Ultrasonography is an accurate and sensitive modality in the evaluation of the tendons around the ankle joint and can be used either as a primary tool of investigation or as a complementary tool with MRI and even in some cases may be used as a final method of diagnosis without the need for the futility of repeating both techniques.

\section{References:}

1. Mosher T, Kransdorf M, Adler R et al. (2015): ACR Appropriateness Criteria acute trauma to the ankle. J Am Coll Radiol., 12(3):221-7.

2. Andrea Gervasio, Paola Bollani, Aurelio Biasio. (2014). US in mid-portion Achilles tendon injury. Journal of Ultrasound, 17 (2):135.

3. Abate M, Silbernagel KG, Salini V, Werner S, Paganelli R et al. (2009). Pathogenesis of tendinopathies: inflammation or degeneration? Arthritis Res Ther. 2009;11(3):235. doi: 10.1186/ar2723. Epub 2009 Jun 30. PMID: 19591655; PMCID: PMC2714139.

4. Klatte-Schulz F, Minkwitz S, Schmock A, Bormann N, Manegold S et al. (2018). Different Achilles Tendon Pathologies Show Distinct Histological and Molecular Characteristics.
International Journal of Molecular Sciences. 2018; 19(2):404. https://doi.org/10.3390/ijms19020404.

5. Hodgson RJ, O'Connor PJ, Grainger AJ. (2012). Tendon and ligament imaging. $\mathrm{Br} \mathrm{J}$ Radiol. 2012;85(1016):1157-1172. doi:10.1259/bjr/34786470.

6. Tam KF, Lui TH. MR Imaging of the Achilles Tendon after Surgical Repair. Open Orthop J. 2017 Jul 31;11:697-703. doi: 10.2174/1874325001711010697. PMID: 28979583; PMCID: PMC5620401.

7. Bianchi S, Martinoli C, De Gautard R, Meyer JM et al. (2005). Ultrasound of the ankle: anatomy of the tendons, bursae, and ligaments. Semin Musculoskelet Radiol. 2005 Sep;9(3):243-59. doi: 10.1055/s-2005-921943. PMID: 16247724.

8. El-Liethy N, Kamal H. (2016). High-resolution ultrasonography and magnetic resonance imaging in evaluation of tendo-ligamentous injuries around ankle joint. The Egyptian journal of radiology and nuclear medicine. (2016) 47: 543-555.

9. Hetta, W. M., \& Niazi, G. (2018). Concordance of US and MRI for diagnosis of ligamentous and tendinous injuries around the ankle. The Egyptian Journal of Radiology and Nuclear Medicine, 49(1), 131-137. https://doi.org/10.1016/j.ejrnm.2017.11.011.

10. Fessell, D. P., \& Jacobson, J. A. (2008). Ultrasound of the Hindfoot and Midfoot. Radiologic Clinics of North America, 46(6), 1027-1043. https://doi.org/10.1016/j.rcl.2008.08.006. 
11. MANSOUR, R., \& JAIN, N. (2013). Imaging of the ankle. Imaging, 22(1), 20110065. https://doi.org/10.1259/imaging.20110065. systematic four-stage approach. World J Orthop. 2016;7(10):657-663. Published 2016 Oct 18. doi:10.5312/wjo.v7.i10.657.

12. Yasui Y, Hannon CP, Hurley E, Kennedy JG.

(2016). Posterior ankle impingement syndrome: A

To cite this article: Hesham M. Farouk, Ahmed H. Saber, Alaa-Eldin A. Mohamed. Role of High Resolution Ultrasound and Magnetic Resonance Imaging In Assessment of the Posterior Ankle Tendons Lesions. BMFJ 2022; 39(Radiology);161-170. DOI: 10.21608/bmfj.2021.88816.1451 